\title{
Longitudinal Current Dissipation in Bose-glass Superconductors
}

\author{
David R. Nelson ${ }^{1}$ and Leo Radzihovsky ${ }^{2}$ \\ Department of Physics, Harvard University, Cambridge, MA $02138^{1}$ \\ Department of Physics, University of Colorado, Boulder, CO $80309^{2}$
}

(October 26, 2018)

\begin{abstract}
A scaling theory of vortex motion in Bose glass superconductors with currents parallel to the common direction of the magnetic field and columnar defects is presented. Above the Bose-glass transition the longitudinal DC resistivity $\rho_{\|}(T) \sim\left(T-T_{B G}\right)^{\nu^{\prime} z^{\prime}}$ vanishes much faster than the corresponding transverse resistivity $\rho_{\perp}(T) \sim\left(T-T_{B G}\right)^{\nu^{\prime}\left(z^{\prime}-2\right)}$, thus reversing the usual anisotropy of electrical transport in the normal state of layered superconductors. In the presence of a current $\mathbf{J}$ at an angle $\theta_{J}$ with the common field and columnar defect axis, the electric field angle $\theta_{E}$ approaches $\pi / 2$ as $T \rightarrow T_{B G}^{+}$. Scaling also predicts the behavior of penetration depths for the AC currents as $T \rightarrow T_{B G}^{-}$, and implies a jump discontinuity at $T_{B G}$ in the superfluid density describing transport parallel to the columns.
\end{abstract}

Recently there have been many efforts to understand the nature of fprtex states and dissipation in disordered high temperature superconductors. These efforts have led to prediction 3 目 that the linear resistivity does in fact vanish at a finite transition temperature to a glassy vortex state, in contrast to the traditional Anderson-Kim picture which always admits small but finite linear resistivity. There is now general agreement on the possibility of a true linearly dissipationless vortex state, and the theory continues in a state of active development.

Pinning in superconductors comes in the form of point disorder such as oxygen vacancies and interstitials as well as correlated disorder such as screw dislocations, twin planes and artificially introduced columnar defects. It was originally proposed that point-like disorder would lead to a vortex glass phase, while the theory in the presence of columnar defects (correlated disorder) predicted an anisotropic "Bose glass" phase, 3 so called because of an analogy with the theory of bosons in superfluids on disordered substrates. 1 Although the general phenomena of divergent pinning barriers for vanishing currents underpins both the vortex glass and the Bose glass theories, the two theories can and have been qualitatively distinguished experimentally via their predictions for the transverse field $H_{\perp}$ response i.e. tilting of the applied magnetic field. While the vortex glass hypothesis predicts isotropic response functions that are nonsingular as $H_{\perp} \rightarrow 0$, Bose glass theory predicts a transverse Meissner effect, with a divergent tilt modulus $c_{44}$ and a cusp-like phase boundary in the $T-H_{\perp}$ phase diagram 3 . More recently the very existence of the threedimensional vortex glass phase has been called into question, by computer simulations with finite screening, l $_{\text {and }}$ by experiments that find a first-order transition in the detwinned samples 1 removing the natural source of correlated disorder. Moreover, experiments that use electron irradiation to inject point centers in sufficient quantities to destroy this first order transition find no evidence for a sharp phase transition with universal exponents. Nevertheless, establishing whether the correlated or point disorder controls the low temperature physics in a given sample remains an open and important question.

Most experimental work on glassy vortex states has focussed on current transport perpendicular to the magnetif field and in the case of Bose glass, perpendicular to the columnar defect axis. An exception is the work by Seow et. al.9, which measures electrical transport parallel to the field direction in $\mathrm{Bi}_{2} \mathrm{Sr}_{2} \mathrm{CaCu}_{2} \mathrm{O}_{8}$ single crystals, irradiated with heavy ions to produce columnar defects, also along the field direction. In this note we analyze the dissipation in the Bose glass superconductor, generalizing the scaling theory to include both longitudinal and transverse currents. Thus, measurements in the simultaneous presence of both longitudinal and transverse currents also provide a clear qualitative distinction between the vortex glass and Bose glass scenarios. When the theory is applied to AC conductivity below $T_{B G}$, we find finite penetration depths parallel and perpendicular to the columns. Scaling predicts a discontinuous jump to zero of the condensate superfluid density describing transport parallel to the columns as $T \rightarrow T_{B G}^{-}$.

We assume point disorder can be neglected at high temperatures and consider a current $J$ at a finite angle $\theta_{J}$ with the $\|$-axis defined by the columnar defects and the magnetic field $B$. Following the usual assumption of a scaling theory that near a continuous transition the diverging correlation length is the only important length scale we determines the temperature dependence and the relation between all the physical quantities and in particular the IV characteristics. Near a Bose glass transition dominated by columnar defects there are two divergent correlation lengths $l_{\perp} \sim\left|T-T_{B G}\right|^{-\nu^{\prime}}$ and $l_{\|} \sim\left|T-T_{B G}\right|^{-\nu_{||}^{\prime}}$ and a correlation time $\tau \sim l_{\perp}^{z^{\prime}} \sim\left|T-T_{B G}\right|^{-z^{\prime} \nu^{\prime}}$, where withip the Bose glass phase $l_{\perp}$ and $l_{\|}$measure the corresponding localization lengths of the vortex lines. Following Ref.B dimensional analysis allows us to relate physical quantities to these correlations lengths. In three dimensions the free energy density scales as $f \sim 1 /\left(l_{\perp}^{2} l_{\|}\right)$. Analogously gauge invariance of the Ginzburg-Landau theory implies that the 
fluctuating vector potential scales according to

$$
\begin{aligned}
& A_{\perp} \sim \frac{1}{l_{\perp}(T)}, \\
& A_{\|} \sim \frac{1}{l_{\|}(T)} .
\end{aligned}
$$

The definitions of the current $\mathbf{J}=\partial f / \partial \mathbf{A}$ and the electric field $\mathbf{E}=-\partial \mathbf{A} / \partial t$ and Eqs.1 allow us also to express $\mathbf{J}$ and $\mathbf{E}$ in terms of correlations lengths and time,

$$
\begin{aligned}
& J_{\perp} \sim \frac{1}{l_{\perp} l_{\|}}, \\
& J_{\|} \sim \frac{1}{l_{\perp}^{2}}
\end{aligned}
$$

and

$$
\begin{aligned}
& E_{\perp} \sim \frac{1}{l_{\perp}^{1+z^{\prime}}} \\
& E_{\|} \sim \frac{1}{l_{\|} l_{\perp}^{z^{\prime}}}
\end{aligned}
$$

where the relation between the correlation time and length $\tau \sim l_{\perp}^{z^{\prime}}$ was used. Given the above dependences of $\mathbf{E}$ and $\mathbf{J}$ we can construct a relation between them, the IV curve, by equating the appropriate dimensionless quantities. Upon first considering separately currents parallel and perpendicular to the field direction, we have

$$
\begin{aligned}
E_{\perp} l_{\perp}^{1+z^{\prime}} & \sim F_{ \pm}^{\perp}\left(l_{\perp} l_{\|} J_{\perp} \phi_{o} / c T\right) \\
E_{\|} l_{\|} l_{\perp}^{z^{\prime}} & \sim F_{ \pm}^{\|}\left(l_{\perp}^{2} J_{\|} \phi_{o} / c T\right)
\end{aligned}
$$

where $\phi_{0}=2 \pi \hbar c / 2 e$ is the flux quantum and we have set $k_{B}=1$. The dimensionless arguments of the scaling functions $F_{ \pm}^{\perp}$ and $F_{ \pm}^{\|}$are the ratios of the work done by the corresponding current to depin the vortex line from the columnar defect to the thermal energy. The difference in the arguments can be understood microscopically. For a transverse current $J_{\perp}$ dissipation arises due the vortex line depinning which proceeds via a "tongue" of a typical area $l_{\perp} \times l_{\|}$lying in the $z-r_{\perp}$-plane. In contrast for a longitudinal current $J_{\|}$the dissipation is due to depinning of vortex helices whose projections span a typical area $l_{\perp}^{2}$ lying in the $\mathbf{r}_{\perp}$-plane.

The scaling functions above $F_{+}$and below $F_{-}$the transition are very different. For $T>T_{B G}$ we expect linear resistivities $E_{\perp}=\rho_{\perp} J_{\perp}$ and $E_{\|}=\rho_{\|} J_{\|}$characteristic of a normal metal. It follows that the positive branches of these scaling functions must vanish linearly, $F_{+}(x) \sim x$, i.e.

$$
\begin{aligned}
& \rho_{\perp} \sim l_{\|} / l_{\perp}^{z^{\prime}}, \\
& \rho_{\|} \sim 1 /\left(l_{\perp}^{z^{\prime}-2} l_{\|}\right) .
\end{aligned}
$$

There is excellent theoreticalt 3 and numerica 1011 evidence that vortices in the liquid phase (i.e. the "superfluid" state of the bosons) "diffuse" as they wanderalong the average field direction. This implies an important relation between the localization lengths near Bose glass 3 transition $l_{\|} \approx\left(T B^{2} / c_{11} \phi_{o}^{2}\right) l_{\perp}^{2}$, where $c_{11} \approx B^{2} / 8 \pi\left(B>>H_{c 1}\right)$ is the bulk modulus of the vortex liquid. Using these relations together with the temperature dependence of $l_{\perp}$ in Eqs. 5 we find,

$$
\begin{aligned}
\rho_{\perp}(T) & \sim\left|T-T_{B G}\right|^{\nu^{\prime}\left(z^{\prime}-2\right)}, \\
\rho_{\|}(T) & \sim\left|T-T_{B G}\right|^{\nu^{\prime} z^{\prime}} .
\end{aligned}
$$

Close to the transition, $\rho_{\|}<<\rho_{\perp}$, which is opposite to the usual normal state resistivity anisotropy in layered superconductors.

Consider a current $\mathbf{J}$ at an angle $\theta_{J}$ with the B field and columnar defect axis. There is now a matrix relating $\mathbf{E}$ to $\mathbf{J}$,

$$
\left[\begin{array}{c}
E_{\perp} \\
E_{\|}
\end{array}\right] \approx\left[\begin{array}{cc}
\rho_{\perp} & 0 \\
0 & \rho_{\|}
\end{array}\right]\left[\begin{array}{c}
J_{\perp} \\
J_{\|}
\end{array}\right]
$$


where the off-diagonal elements are zero if we neglect the very small and poorly understood Hall effect. The electric field $\mathbf{E}$ (in this single parameter scaling theory) will be at a temperature-dependent angle $\theta_{E}(T)=\tan ^{-1}\left(E_{\perp} / E_{\|}\right)$, given by

$$
\tan \left(\theta_{E}\right) \propto \tan \left(\theta_{J}\right) /\left(T-T_{B G}\right)^{2 \nu^{\prime}}
$$

where $\tan \left(\theta_{J}\right)=J_{\perp} / J_{\|}$. Equation 8 predicts that near $T_{B G}$ the angle $\theta_{E}$ for Bose glass superconductor has a universal tempgrature dependence controlled by the Bose glass transverse localization length exponent $\nu^{\prime}$, estimated to be $\nu^{\prime} \approx 1.19$ Besides providing a direct measurement of $\nu^{\prime}$, Eq.8 predicts the electric field direction $\theta_{E}(T \rightarrow$ $\left.T_{B G}^{+}\right) \approx \pi / 2-\left(T-T_{B G}\right)^{2 \nu^{\prime}} \cot \left(\theta_{J}\right) \rightarrow \pi / 2$, for any current direction $\theta_{J} \neq 0$, independent of microscopic details such as the intrinsic resistivity anisotropy of the normal state. Because vortex glass dissipation is isotropic (aside from the intrinsic material anisotropy) the corresponding expression for vortex glass predicts a $\theta_{E}$ that is asymptotically temperature independent as $T \rightarrow T_{V G}$ and depends continuously on the direction of the current $\theta_{J}$.

The significantly faster vanishing of longitudinal resistivity, predicted by Eq.6 as $T \rightarrow T_{B G}$ has already been observed in recent experiments by Seow, et al.9, which finds $\nu^{\prime} z^{\prime}=8.5 \pm 1.6$, consistent with other estimates of $\nu^{\prime}=12111$ and $z^{\prime}=6.0 \pm 0.511$. However, as is evident from Eq.8, an additional check on the Bose glass theory can be made by testing to see if $\lim _{T \rightarrow T_{B G}^{+}} \theta_{E}(T)=\pi / 2$ for any $\theta_{J} \neq 0$. Equivalently, the measurement of the vanishing ratio $\rho_{\|}(T) / \rho_{\perp}(T)$ as $T \rightarrow T_{B G}$ allows a direct determination of $\nu^{\prime}$.

The scaling Eq. 1 predicts nonlinear IV characteristics at the Bose glass transition, $T=T_{B G}$. 2. that there is a well defined IV characteristics demands that the divergent correlation lengths cancel on both sides of these equations, which can only be satisfied by a specific power-law behavior of $F^{\perp}(x)$ and $F^{\|}(x)$ as $x \rightarrow \infty$, leading to

$$
\begin{aligned}
E_{\perp}\left(J_{\perp}\right) & \sim J_{\perp}^{\left(1+z^{\prime}\right) / 3}, \\
E_{\|}\left(J_{\|}\right) & \sim J_{\|}^{\left(2+z^{\prime}\right) / 2} .
\end{aligned}
$$

The longitudinal dissipation is thus weaker and more nonlinear than the transverse one.

Below the Bose glass transition the dissipation is highly nonlinear and is characterized by potential barriers that diverge in the limit of vanishing current,

$$
\begin{aligned}
E_{\perp}\left(J_{\perp}\right) & \sim e^{-\left(J_{\perp}^{o} / J_{\perp}\right)^{\mu} \perp} \\
E_{\|}\left(J_{\|}\right) & \sim e^{-\left(J_{\|}^{o} / J_{\|}\right)^{\mu} \|},
\end{aligned}
$$

where $\mu_{\perp} \rightarrow 1 / 3$ as $J_{\perp} \rightarrow 0$ in bulk samples/ and the calculation in Sec.II-E of Ref.B suggests that $\mu_{\|}=1$.

The scaling theory can be further generalized to a finite frequency $\omega$ by an addition to the scaling functions in Eqs. 4 of another dimensionless variable $l_{1}^{\prime} z^{\prime}$. At finite frequency there is linear dissipation at all finite temperatures characterized by linear conductivities 32.23

$$
\begin{aligned}
\sigma_{\perp}(\omega, T) & \sim l_{\perp}^{z^{\prime}-2} f_{ \pm}^{\perp}\left(\omega l_{\perp}^{z^{\prime}}\right), \\
\sigma_{\|}(\omega, T) & \sim l_{\perp}^{z^{\prime}} f_{ \pm}^{\|}\left(\omega l_{\perp}^{z^{\prime}}\right) .
\end{aligned}
$$

Requiring that the conductivities are finite at the Bose glass transition, the scaling theory together with the KramersKronig relation lead to

$$
\begin{aligned}
\sigma_{\perp}\left(\omega, T_{B G}\right) & \sim\left(\frac{1}{-i \omega}\right)^{1-2 / z^{\prime}}, \\
\sigma_{\|}\left(\omega, T_{B G}\right) & \sim \frac{1}{-i \omega}
\end{aligned}
$$

predicting a universal phase lag between current and voltage, which in the case of $\sigma_{\|}$is $\pi / 2$, independent of critical exponents. For $T<T_{B G}, \sigma_{\perp, \|} \sim n_{s}^{\perp, \|} /(-i \omega)$, implying scaling for the superfluid number densities describing charge transport by Cooper pairs perpendicular and parallel to the columns,

$$
\begin{aligned}
n_{s}^{\perp} & \sim 1 / l_{\|} \sim 1 / l_{\perp}^{2}, \\
n_{s}^{\|} & \sim l_{\|} / l_{\perp}^{2}=\mathrm{constant},
\end{aligned}
$$


as $T \rightarrow T_{B G}^{-}$, consistent with the corresponding Josephson relations for superfluid densities in an anisotropic superconductor.

More precisely, for $n_{s}^{\|}$we expect the relationship

$$
\lim _{T \rightarrow T_{B G}^{-}} n_{s}^{\|}(T)=\lim _{T \rightarrow T_{B G}^{-}} \frac{m T}{\hbar^{2}} \frac{l_{\|}(T)}{l_{\perp}^{2}(T)}=\text { constant },
$$

where we take $m$ to be the mass of a Cooper pair, and $l_{\|}(T)$ and $l_{\perp}(T)$ are defined in the usual way in terms of the decay of the transverse BCS order parameter correlation function 14 . We have assumed in the spirit of scaling that $l_{\|}(T)$ and $l_{\perp}(T)$ are the only diverging length scales near $T_{B G}$. The lengths $l_{\|}(T)$ and $l_{\perp}(T)$ must then diverge as $T \rightarrow T_{B G}^{-}$in the same way as the corresponding correlation lengths above $T_{B G}$. Since $\lim _{T \rightarrow T_{B G}^{+}} l_{\|}(T) / l_{\perp}^{2}(T) \approx$ $T_{B G} B^{2} d f_{11} \phi_{0}^{2}=$ constant, required by finiteness of the boson compressibility $c_{11}$ (vortex line compression modulus) at $T_{B G}$ 进, we are led to Eq.14. In the likely event that for short range interaction both superfluid densities vanish in the vortex liquid state for $T>T_{B G}$, our analysis therefore implies a striking result: In contrast to $n_{s}^{\perp}$ which vanishes smoothly as $T \rightarrow T_{B G}^{-}$(similar to a conventional superconductor), $n_{s}^{\|}$has a jump discontinuity at $T=T_{B G}$ analogous to a stiffness of a system at a Kosterlitz-Thouless transition. The $n_{s}^{\|}$jump discontinuity is consistent with Eq.12]b, predicting that $\sigma_{\|}$'s $\omega$ dependences at and below $T_{B G}$ are identical.

Using above results for the $\mathrm{AC}$ conductivities together with Maxwell's equations, we find the effective penetration lengths $\lambda_{\text {eff }} \sim 1 / \sqrt{\omega \mid \sigma(\omega)} \mid$ for the AC currents $J_{\perp}$ and $J_{\|}($for $\omega \rightarrow 0)$ to be $\tilde{\lambda}_{\perp} \sim \sqrt{l}_{\|} \sim l_{\perp}$ and $\tilde{\lambda}_{\|} \sim l_{\perp}^{2} / l_{\|}=$ constant, respectively. While $\tilde{\lambda}_{\perp}$ diverges as $T \rightarrow T_{B G}^{-}, \tilde{\lambda}_{\|}$remains finite at the transition and discontinuously jumps to infinity for $T>T_{B G}$.

The scaling theory for longitudinal currents can be further generalized to include the response to the transverse magnetic field $H_{\perp}$, previously analyzed for $J_{\perp}$ in Ref 3 . For simplicity assuming purely longitudinal current, $E_{\|}$from Eq. - becomes

$$
E_{||} l_{\|} l_{\perp}^{z^{\prime}} \sim F_{ \pm}^{\|}\left(l_{\perp}^{2} J_{\|} \phi_{o} / c T, H_{\perp} l_{\perp} l_{\|} / \phi_{0}\right)
$$

which by arguments similar to above predicts a cusp-like phase boundary in the $\mathrm{T}-\mathrm{H}_{\perp}$-plane between the Bose glass where $\rho_{\|}\left(H_{\perp}<H_{\perp}^{c}(T)\right)=0$ and the vortex liquid phase with $\rho_{\|}\left(H_{\perp}>H_{\perp}^{c}(T)\right)>0$. This boundary, given by

$$
H_{\perp}^{c}(T) \sim \pm\left|T-T_{B G}\right|^{3 \nu^{\prime}},
$$

is consistently identical to the phase boundary obtained based on the criterion of the vanishing of the transverse resistivity $\rho_{\perp}(T)$, as must be the case if there is a single transition to the Bose glass phase.

Equation 15 can also be used to predict how $\rho_{\|}\left(H_{\perp}\right)$ vanishes as $H_{\perp} \rightarrow 0$, with $T=T_{B G}$,

$$
\rho_{\|}\left(T=T_{B G}, H_{\perp}\right) \sim\left|H_{\perp}\right|^{z^{\prime} / 3} .
$$

This result is to be contrasted with the more slowly vanishing transverse linear resistivity $\rho_{\perp}\left(T=T_{B G}, H_{\perp}\right) \sim$ $\left|H_{\perp}\right|^{\left(z^{\prime}-2\right) / 3}$ found in Ref.B.

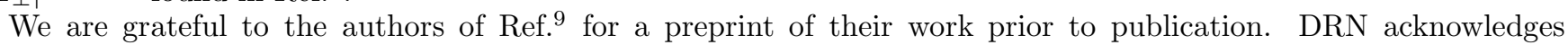
financial support by National Science Foundation, in part by the MRSEC program through Grant DMR-9400396 and through Grant DMR-9417047. LR was supported by the National Science Foundation CAREER award, through Grant DMR-9625111.

\footnotetext{
${ }^{1}$ G. Blatter et. al., Rev. Mod. Phys. 66, 1125 (1994); D. Huse and L. Radzihovsky, in Proceedings of 1993 Altenberg Summer School, Fundamental Problems in Statistical Mechanics VIII, edited by H. van Beijeren and M. H. Ernst (Elsevier, Netherlands); D. R. Nelson, in Phenomenology and Applications of High-Temperature Superconductors, edited by K. Bedell, et. al. (Addison-Wesley, Reading MA).

${ }^{2}$ M. P. A. Fisher, Phys. Rev. Lett 62, 1415 (1989). D. S. Fisher, M. P. A. Fisher, and D. A. Huse, Phys. Rev. B43, 130 (1991)

${ }^{3}$ D. R. Nelson and V. M. Vinokur, Phys. Rev. Lett. 68, 2398 (1992); Phys. Rev. B. 48, 13060 (1993).

${ }^{4}$ M. P. A. Fisher, P. B. Weichman, G. Grinstein, and D. S. Fisher, Phys. Rev. B 40, 546 (1989).
} 
${ }^{5}$ C. Wengel and A. P. Young, cond-mat/9605087 preprint (1996).

${ }^{6}$ H. Safar, et. al., Phys. Rev. Lett. 69, 824 (1992); W. K. Kwok, et. al., Phys. Rev. Lett. 69, 3370 (1992).

7 J. A. Fendrich, et. al. Phys. Rev. Lett. 74, 1210 (1995).

${ }^{8}$ We do not consider here a third possible low temperature phase, the "Bragg glass", which is a topologically perfect Abrikosov flux lattice pinned by point disorder. See T. Giamarchi and P. Le Doussal, Phys. Rev. B 52, 1242 (1995), and M. J. P. Gingras and D. A. Huse, cond-mat/9601123 preprint (1996).

${ }^{9}$ W. S. Seow, R. A. Doyle, A. M. Campbell, G. Balakrishnan, K. Kadowaki, and G. Wirth, Phys. Rev. B (in press).

${ }^{10}$ W. Krauth, T. Trivedi, and D. Ceperley, Phys. Rev. Lett. 672307 (1991); E. S. Sorensen, et. al. ibid 69828 (1992).

${ }^{11}$ M. Wallin and S. Girvin, Phys. Rev. B 47, 14642 (1993).

12 A. T. Dorsey, Phys. Rev. B 43, 7575 (1991).

${ }^{13}$ W. Jiang, et al., Phys. Rev. Lett. 72, 550 (1994).

${ }^{14}$ See, e.g., P. C. Hohenberg, A. Aharony, B. I. Halperin, and E. D. Siggia, Phys. Rev. B 13, 2986 (1976). Here, the phase fluctuations in the BCS order parameter are assumed to be described by an anisotropic free energy $\delta F \equiv$ $\frac{1}{2} \int d^{3} r\left[\frac{\hbar^{2}}{m} n_{s}^{\|}\left(\partial_{z} \theta_{B C S}-\frac{2 \pi}{\phi_{0}} A_{z}\right)^{2}+\frac{\hbar^{2}}{m} n_{s}^{\perp}\left(\nabla_{\perp} \theta_{B C S}-\frac{2 \pi}{\phi_{0}} \mathbf{A}_{\perp}\right)^{2}\right]$, where we take $m$ to be the mass of a Cooper pair in this definition of $n_{s}^{\|, \perp}$. The BCS phase $\theta_{B C S}$ and the gauge field $\mathbf{A}$ are measured relative to the corresponding random background fields determined by a quenched set of vortex lines trapped on columnar pins. This free energy is a coarse-grained description valid on scales large compared to the vortex spacing. 